\title{
STORIE DI PAROLE ED ETIMI DEL DIALETTO TRIESTINO
}

\section{Triest. mod. mocadòr "fazzoletto" e triest. ant. mocadòr "spegnitoio"}

Credo meriti soffermarsi per un po' su una vecchia coppia di omonimi del dialetto triestino, mocadòr "fazzoletto" e mocadòr "spegnitoio".

Il termine mocadòr "fazzoletto", che può considerarsi caratterizzante del dialetto triestino moderno è stato trattato, nel GDDT, abbastanza esaurientemente, in quanto che risultano correttamente messe in rilievo le concordanze lessicali più significative, necessarie per arrivar a tracciare la sua storia e fissarne l'etimo. Ma mentre questo risulta praticamente assicurato (lat. volg. *MUCCĀRE "soffiarsi il naso"), le vicende per cui si è arrivati a triest. mocadòr "fazzoletto" restano incerte: direttamente dalla base MUCCĀRE, attraverso una trafila fonetica locale, di stampo italo-settentrionale? una rielaborazione, pure locale, della voce veneziana mocaòr (significante anch'esso "fazzoletto" v. Boerio, e non solo "spegnitoio", come pare affermare il Doria)? oppure prestito dallo spagnolo mocador "id.", non si sa attraverso quali canali? A queste tre alternative è possibile, ora, aggiungere una quarta, in quanto che si intravede, causa esigenze cronologiche, la possibilità, come sostenuto dal DEDI, di un prestito dal catalano, possibile poiché il tipo mocadòr, moccatore risulta attestato anche in sardo (cfr. Wagner DES s.v.) e nel napoletano e altri dialetti meridionali. Il tipo mucaturi è infatti forma certamente rifatta su un più antico mucaduri, con sostituzione del suffisso contenente - $d$ - intervocalica con altro contenente un $-t$ - secondario, ossia - $d$ - "meridionalizzato". (A questo proposito si avverte però che a Napoli, centro di diffusione del lessema, una forma con - $d$ - intervocalico conservato non è mai esistita).

Sennonché a queste "storie" mancavano ancora alcuni tasselli che ora mi premuro di inserire nell'elenco dei dati già a nostra disposizione.

1. mocadòr è forma anche gergale, trovandosi attestata come tale a Bologna. Ce ne aveva informato Aldo Menarini, fin dal 1942, in un suo noto lavoro ${ }^{1}$.

2. mocadòr ha varcato l'Adriatico in un punto solo, a Trieste, e di là si è irradiato a Capodistria (Manzini-Rocchi s.v.). Se Capodistria, dove si parla un dialetto istroveneto, lo avesse tolto a Venezia, avrebbe accettato la forma, appunto veneziana, mocà̀r e non mocadòr.

Combinando questi due dati e la constatazione che la base MUCCĀRE è tipica dell'Italia settentrionale, si perverrebbe alla conclusione che mocadòr ha la sua origine quasi certamente in questa zona della Romania, non altrove (Meridione, penisola iberica). Come a dire, per concludere, che il triestino mocadòr è più autonomo

1 A. Menarini, I gerghi bolognesi, Modena 1942. 
di quanto si potrebbe credere a prima vista e svincolato dal parallelo veneziano mocaòr. Ed è un vero peccato che non risultino attestate forme del genere per le zone di trapasso come Grado, Marano Lagunare e Bisiaccaria ${ }^{2}$. In tal caso detta conclusione sarebbe matematicamente sicura.

Per quanto riguarda l'altro mocadòr "spegnitoio, smorzacandele" dirò subito che esso non ha nulla a che fare con mocadòr "fazzoletto". Infatti esso si trova attestato in un inventario del 1631 nella sezione dedicata ad attrezzi da cucina". Quindi "spegnitoio" o simm., solo casualmente omofono a mocadòr "fazzoletto", e avente in comune con esso solo l'etimo "lontano" (MUCCĀRE), ma per il resto, ossia per la sua storia e i suoi successivi accostamenti, del tutto indipendente ${ }^{4}$ : un'altra parola, a dirla in breve.

Preciserò anche che detta omonimia, ossia la contemporanea presenza, nel dialetto, dei due mocadòr sarà stata certo presente nella Trieste del XVII secolo, ma in epoca successiva totalmente rimossa, in quanto che il secondo di questi mocadòr fu sostituito dal termine distudacandèle, certamente più efficace, anche per una certa sua innata trasparenza.

\section{2. triest. rùcola "ruffiana"}

Non so se c'è ancora qualcuno a Trieste che adoperi questa caratteristica parola, sia pur limitatamente al fraseologico far de rùcola "reggere il lume (o il moccolo), far da ruffiana". Anche qui ci troviamo di fronte ad una vistosa omonimia: nel dialetto rùcola significa, com'è noto, anche "ruchetta", pianta selvatica aromatica, che si mangia assieme al radicchio. E' evidente che questa non è che un omofono, in ultima analisi, un italianismo, a sua volta dal latino ERUCA "id." (su altri particolari v. GDDT s.v. rùcola). La nostra rùcola "ruffiana" a dire il vero attestata fin dal 1877 sotto la variante rùcola nel primo Kosovitz (E. Kosovitz, Dizionario-vocabolario del dialetto triestino e della lingua italiana, Trieste), è, invece, assai oscura, si direbbe quasi isolata (così il GDDT s.v.), a prescindere dal suo riapparire in una limitata striscia di territorio toscano (Livorno, Lucca, Pistoia, Prato), dove essa compare nuovamente con questo singolare significato. E forse, proprio per questo suo isolamento semantico sono propensa a credere, nuovamente, a voce gergale, ossia ad una creazione scherzosa basata sul verbo tedesco rücken "spingere" e anche "spacciare, offrire in vendita". L'evoluzione del significato è, sorprendentemente, parallela a quella, ben nota, accertata per il verbo inglese to push, anch'esso gergale, quando significa "vendere, spacciare (droga o

2 Ricorderò che mucadòr e termini affini sono del tutto estranei al friulano.

3 '"....] doi lavezi di bronzo, un para di mollette, un mocador, una dozina di vasi col manigo" (P. Covre, L'arredamento di una casa triestina del '600, in Folclore Giuliano, Atti del III Convegno Trieste 14-15 novembre 1998, Istituto Giuliano di Storia, Cultura e Documentazione Trieste 2001, p.173)

4 Ad es. come friul. smociadôr (Faggin s.v.) "id.", assai rilevante, una volta accertato che mocadòr "fazzoletto" è privo di corrispondenze in quest'area dialettale, oppure istroven. mocàr "smorzare, smoccolare (la candela)" (istrioto mucâ), Rosamani ss.vv. 
erba)", da cui poi il nomen agentis pusher "spacciatore" (cfr. su quest'ultimo G. Borton, in "Panorama" 26-2-2001 p. 15).

Questa vicenda ci insegnerebbe, oltretutto, che i tedeschismi del dialetto triestino non sono tutti calati dal Nord ma hanno seguito, talora, vie di penetrazione più tortuose.

\author{
Bibliografia - Abbreviazioni impiegate \\ BOERIO \\ G. Boerio, Dizionario del dialetto veneziano ${ }^{2}$, Venezia 1856. \\ DEDI \\ M. Cortelazzo - C. Marcato, I dialetti italiani. Dizionario etimologico, Torino 1998. \\ FAGGIN \\ GDDT \\ Giorgio Faggin, Vocabolario della lingua friulana, 2 voll., Udine 1988. \\ M. Doria - C. Noliani, Grande dizionario,storico, fraseologico, etimologico del dialetto triestino, Trieste 1987. \\ MANZINI-ROCCHI \\ Giulio Manzini - Rocchi, Dizionario storico fraseologico etimologico del dialetto di Capodistria, Trieste- \\ Rovigno 1995. \\ PANORAMA \\ "Panorama" settimanale, Mondadori, Milano. \\ ROSAMANI \\ Enrico Rosamani, Vocabolario giuliano, Bologna 1958. \\ WAGNER DES \\ Max Leopold Wagner, Dizionario etimologico sardo, Heidelberg 1957-1962.
}

Povzetek

ZGODOVINA IN ETIMOLOGIJA DVEH BESED IZ TRIESTINSKEGA GOVORA

$\mathrm{V}$ modernem triestinskem govoru (s čimer razumemo sodobno beneško narečje $\mathrm{v}$ Trstu) ima mocadòr 'robec' docela drugačno zgodovino kot enakozvočni mocadòr 'kajfež, ugaševalec za sveče'; dandanes je ta že iz rabe, vendar ga je mogoče dokumentirati v letu 1631. Če upoštevamo, da ga najdemo tudi $\mathrm{v}$ žargonu $\mathrm{v}$ Bologni in $\mathrm{v}$ beneščini kot mocaòr, smemo trditi, da g re za severnoitalijanski izraz in ne morda za iz španščine ali katalonščine prevzeto besedo.

Tudi rùcola 'zvodnica' ima vzporedno, enakozvočno besedo rùcola 'rukvica'. Ta je kot italianizem iz lat. ERUCA SATIVA, ona pa ljudska izpeljanka iz nem. rücken 'poriniti', kar je najbrž zaznavno tudi v pomenu it. spacciare 'razpečavati droge, ipd.' . Enak pomenski premik najdemo $v$ angl. to push/pusher. 